\title{
First-principles theory of metal-alkaline earth oxide interfaces
}

\author{
Matías Nuñez ${ }^{1}$ and Marco Buongiorno Nardelli ${ }^{1,2}$ \\ ${ }^{1}$ Center for High Performance Simulation and Department of Physics, North Carolina State University, \\ Raleigh, North Carolina 27695, USA \\ ${ }^{2}$ Computer Science and Mathematics Division, Oak Ridge National Laboratory, Oak Ridge, Tennessee 37831-6359, USA
}

(Received 27 March 2006; published 21 June 2006)

\begin{abstract}
Using calculations from first principles, we discuss the interplay between structure and functionality at metal-insulator interfaces using the paradigmatic example of the junctions between various metals $(\mathrm{Ag}, \mathrm{Pd}, \mathrm{Pt}$, $\mathrm{Ni}, \mathrm{Cu}, \mathrm{Al})$ and binary alkaline earth crystalline oxides $(\mathrm{BaO}, \mathrm{CaO}$, and $\mathrm{SrO})$. Our results demonstrate that it is possible to tune the Schottky barrier height in a very broad range of values by manipulating the metal at the interface, and elucidate the role of the relative overlap in the density of states of the different components in determining the band alignment. We conclude by stating a "modified Schottky-Mott rule" for this class of metal-insulator heterojunctions.
\end{abstract}

DOI: $10.1103 /$ PhysRevB.73.235422

PACS number(s): 73.20.At, 68.35.Ct, 71.15.Mb, 73.40.Rw

\section{INTRODUCTION}

Advances in the epitaxial growth of ultrathin films and heterojunctions have made viable new classes of device applications that may sustain the semiconductor roadmap for another decade. However, this progress will require an indepth understanding and utilization of electronic processes at the nanoscale. One of the central issues is the role of interfaces between materials, where most of the electronic properties of the system are determined. In particular, understanding the formation of the Schottky barrier in metal-oxide insulator interfaces (the energetic barrier the electrons have to overcome to go from the valence band of the metal to the conduction band of the oxide) is of paramount importance for the design of metallic gate contacts and the engineering of advanced electronic devices. The ability to tune and control the band alignment at the heterojunction, a fundamental requirement for improving device efficiency, can come only from a detailed knowledge of all the factors that come into play when the two materials are brought together. This is a truly nanoscale problem, where the position of the individual atoms at the interface determines dramatically the electronic properties of the whole system. ${ }^{1}$

In this paper, we will discuss some fundamental ideas on the physics of nanoscale interfaces and the interplay between structure and functionality using the paradigmatic examples of the junctions between metals $(\mathrm{Ag}, \mathrm{Pd}, \mathrm{Pt}, \mathrm{Ni}, \mathrm{Cu}, \mathrm{Al})$ and binary crystalline oxides $(\mathrm{BaO}, \mathrm{CaO}$, and $\mathrm{SrO})$. The paper is divided as follows: after the Introduction (Sec. I) we discuss the main theoretical and methodological techniques in Sec. II, where we also give the details on the geometrical structure of our interfaces; Sec. III contains all our results and discussions: Sec. III A deals with the relationship between geometrical parameters of the interface and the SBH, while in Sec. III B we discuss the results in terms of the electronic properties of the system and propose a modified SchottkyMott rule that applies to this class of heterojunctions. Finally, we give our conclusions in Sec. IV.

\section{INTERFACE GEOMETRY AND COMPUTATIONAL DETAILS}

The geometry of the metal/oxide interface is characterized by two geometrical parameters, the average interface dis- tance between the metal and oxide interfacial planes, and the so-called rumpling parameter, which is a measure of the relative displacement of the atoms in a given plane with respect to the flat bulk geometry (the corrugation of the plane), as shown in Fig. 1, right panel, and described in the figure caption. In particular, we will be mostly concerned with the rumpling on the first layer of atoms in the oxide, where the value of the parameter is proportional to the relative displacement of the metal and oxygen atoms. One of the main

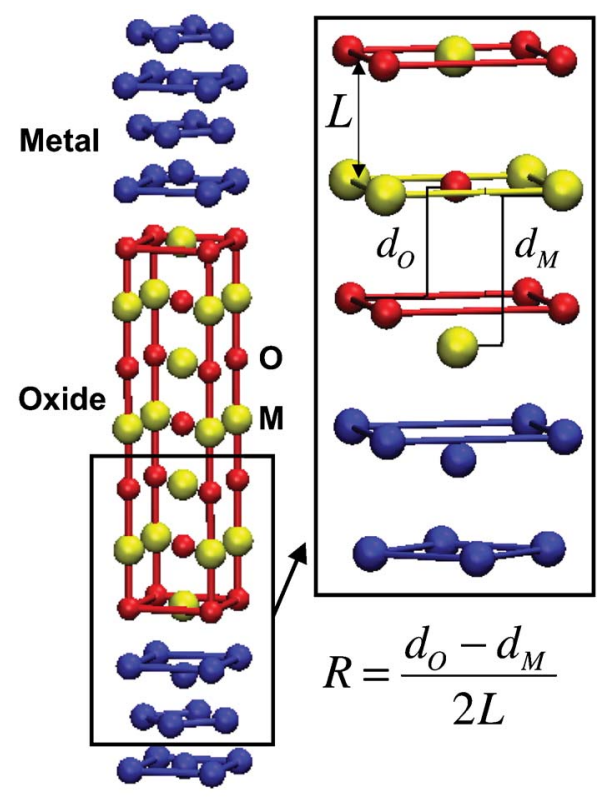

FIG. 1. (Color online) (Left panel) Geometry of the supercell used in the calculation. (Right panel) Definition of the rumpling parameter. The rumpling is a measure of the relative displacement of the atoms in the interface oxide plane. It is an $a$-dimensional parameter given by $R=\left(d_{\mathrm{O}}-d_{\mathrm{M}}\right) / 2 L$, where $d_{\mathrm{O}}$ is the distance between the $\mathrm{O}$ atoms in the first and the second layer of the crystalline oxide, $d_{\mathrm{M}}$ is the distance between the metal atoms (of the oxide) in those same layers, and $L$ is the lattice parameter of the bulk oxide. Note that a positive rumpling corresponds to the $\mathrm{O}$ atoms moving outward (metals moving inwards) and vice-versa for a negative rumpling. 


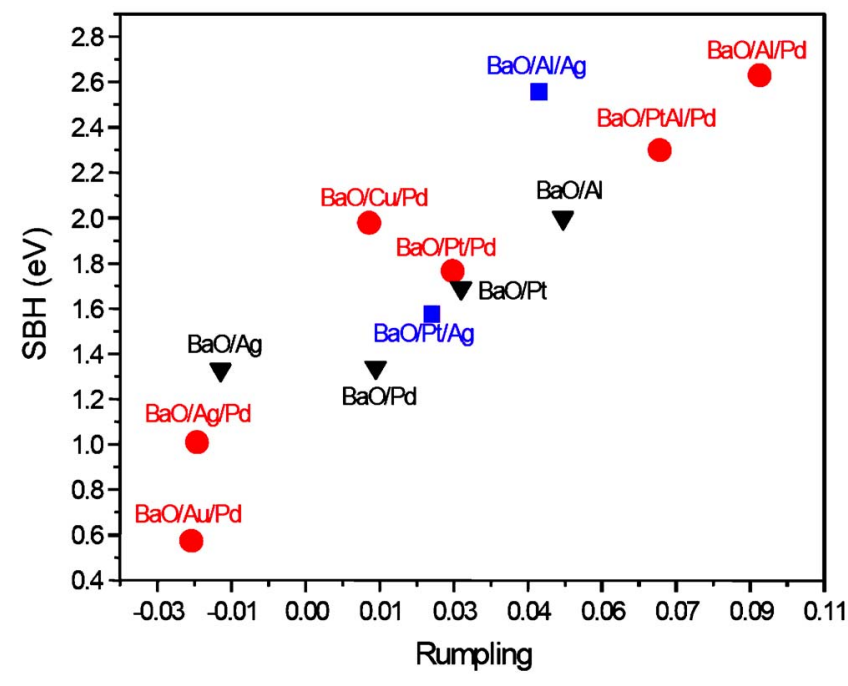

FIG. 2. (Color online) SBH versus rumpling of the first oxide layer at the interface with different metals (black triangles) and metal intralayers [red (gray) circles and blue (dark gray) squares].

conclusions of our study is that this parameter plays a crucial role in determining the electronic properties and the Schottky barrier height $(\mathrm{SBH})$ at the metal/oxide interface and that control of this quantity is equivalent to being able to continuously tune the barrier height in a broad range of values. As an illustration of this concept, in Fig. 2 we show the correlation between the $\mathrm{SBH}$ and the rumpling parameter in interfaces between $\mathrm{BaO}$ and a wide variety of metals $(\mathrm{Ag}, \mathrm{Pd}, \mathrm{Pt}$, $\mathrm{Cu}, \mathrm{Al}$ ), and combination of metal intralayers $(\mathrm{Au} / \mathrm{Pd}, \mathrm{Ag} / \mathrm{Pd}, \mathrm{Cu} / \mathrm{Pd}, \mathrm{Pt} / \mathrm{Pd}, \mathrm{Pt} / \mathrm{Ag}, \mathrm{Al} / \mathrm{Ag})$. As it is clear from the data, there is an almost linear correlation between the SBH and the rumpling at the first layer of the oxide in a range of variation that spans almost the full theoretical band gap of the bulk oxide and points towards the critical role of the nanoscale structure in determining the properties of such interfaces.

All interfaces were modeled with periodically repeated supercells, where the metal and oxide slabs were terminated to have two equivalent junctions along the (001) direction, as in Fig. 1, left panel. To achieve minimum lattice mismatch the $x$ and $y$ axes of the metal are rotated $45^{\circ}$ with respect to the $x$ and $y$ axes of the oxide cell, so that the ratio of the two cubic lattice parameters is equal to $1 / \sqrt{2}$. To further minimize the interfacial strain effect we chose pairs of metals and oxides with lattice mismatches less than $4 \%$. The interfaces chosen for this study are: $\mathrm{SrO} / \mathrm{Cu}, \mathrm{CaO} / \mathrm{Ni}, \mathrm{BaO} / \mathrm{Pd}, \mathrm{BaO} / \mathrm{Pt}, \mathrm{BaO} / \mathrm{Al}, \quad$ and $\mathrm{BaO} / \mathrm{Ag}$ with lattice mismatches of $1.2 \%, 1.5 \%, 0.1 \%$, $1.2 \%, 2.4 \%$, and $3.8 \%$, respectively (see Table I). All oxides crystallize in the $\mathrm{NaCl}$ geometry, while the structure of the metal is fcc cubic. This choice distinguishes our investigation from most of the existing work on metal-oxide interfaces, which focuses on $\mathrm{MgO}$ for the particular catalytic properties of oxide-supported metal clusters ${ }^{2}$ but that suffers of larger instabilities due to large interfacial strain. In the most stable interface configuration, the atoms of the first layer of the oxide slab sit right on top of the atoms of the first
TABLE I. Theoretical lattice parameters and work functions for the bulk systems studied in this work. Oxides lattice parameters are for the rotated cell $\left(a_{0} / \sqrt{2}\right)$ to better display the actual mismatch with the metal cell. Experimental values are indicated in parenthesis when available (Ref.11).

\begin{tabular}{lcc}
\hline \hline & Lattice Parameter $(\AA)$ & Work function $(\mathrm{eV})$ \\
\hline $\mathrm{Ag}$ & $4.01(4.09)$ & $4.75(4.64)$ \\
$\mathrm{Al}$ & $3.95(4.05)$ & $4.42(4.41)$ \\
$\mathrm{Au}$ & $4.05(4.08)$ & $5.49(5.47)$ \\
$\mathrm{Cu}$ & $3.54(3.61)$ & $4.62(4.41)$ \\
$\mathrm{Ni}$ & $3.42(3.52)$ & $5.40(5.22)$ \\
$\mathrm{Pd}$ & $3.87(3.89)$ & $5.52(5.40)$ \\
$\mathrm{Pt}$ & $3.91(3.92)$ & $6.07(5.67)$ \\
$\mathrm{CaO}$ & $3.36(3.40)$ & 5.76 \\
$\mathrm{SrO}$ & $3.58(3.65)$ & 3.92 \\
$\mathrm{BaO}$ & $3.86(3.90)$ & 4.14 \\
\hline \hline
\end{tabular}

layer of the metal in a one to one, cubic-on-cubic correspondence, as displayed in Fig. 1. For all the supercell geometries we used the in plane lattice parameter of the oxide at equilibrium, so that all residual strain is localized in the metal layer. The lattice parameters are obtained performing independent $a b$ initio calculations for the bulk systems are reported in Table I and are in excellent agreement with experimental results. For all the interfaces, we carefully checked that the number of layers considered were enough to reproduce the bulk properties of the two materials far from the interface. In most cases, 7 layers of oxide and 7 layers of metal were found to be sufficient. The calculations were performed using density functional theory (DFT) within the local density approximation (LDA), using ultrasoft pseudopotentials and a plane waves basis set. ${ }^{3}$

The calculation of the SBH follows a well-established procedure: ${ }^{4}$ the barrier height is partitioned into two contributions, the potential lineup across the interface, $\Delta V$, and the band alignment term, $\Delta E$. The last one is the difference between the Fermi energy of the metal and the valance band edge of the oxide, each measured relative to the average electrostatic potential of the corresponding bulk crystal, obtained from independent bulk calculations. The potential lineup instead is an interface specific property, and it is obtained from supercell calculations after the full relaxation of the ions. It is important to note that the SBH that we report here are barriers between the valence band of the oxide and the Fermi energy of the metal, relevant for hole carriers. The corresponding barrier for electrons can be obtained adding to those the energy gap of the insulator. ${ }^{5}$ The errors in the SBH values due to the averaging technique ${ }^{5}$ are of the order of $50 \mathrm{meV}$.

We started our study by looking at the SBH in superlattices of individual metal/oxide systems, and we proceeded with a systematic characterization of the band alignment between the different materials. 


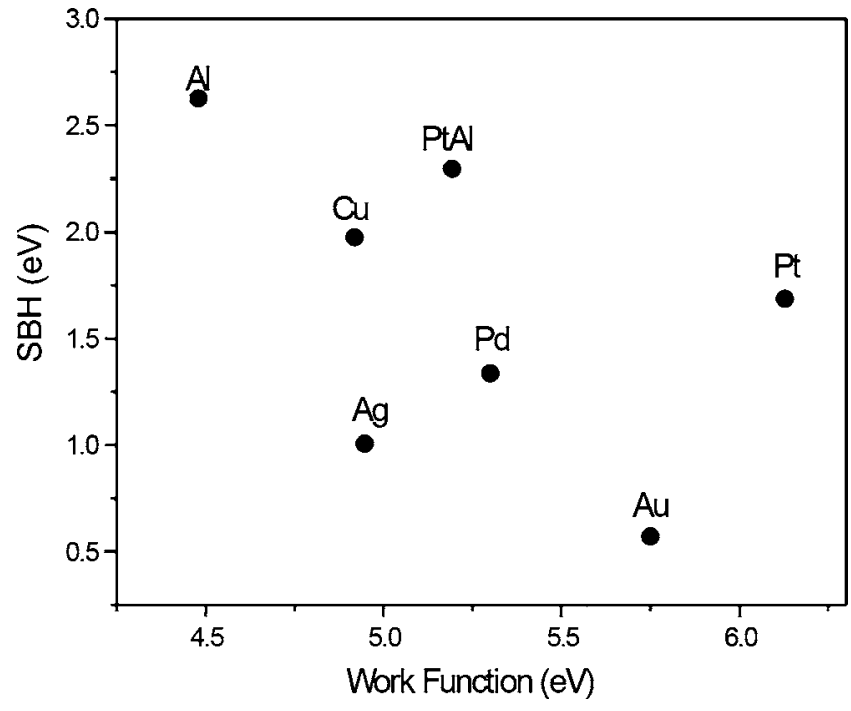

FIG. 3. SBH versus Work Function. The $\mathrm{SBH}$ of a $\mathrm{BaO}$ slab interfaced with different metals vs the work function of these metals. The metals are actually formed by a slab of Pd with a layer of a different metal on top. In this way the work function of the Pd slab can be tuned with a consequent effect on the SBH of the interface $\mathrm{BaO} / \mathrm{METAL} / \mathrm{Pd}$.

\section{RESULTS AND DISCUSSION}

\section{A. Geometrical structure and the SBH}

Traditionally, the electronic properties of a metal/insulator interfaces have been described in terms of the two limits of strong localization and Fermi level pinning by interface states (the Bardeen limit) ${ }^{6}$ and of weak localization and superposition of the bulk properties (the Schottky-Mott ${ }^{7,8}$ limit). Our results for a fixed oxide and a variety of metals summarized in Fig. 2, clearly show that the Fermi energy level varies a lot between the different interfaces and no localized states are observed at the interface. This implies that no Fermi energy pinning is observed and that the Bardeen limit $^{6}$ does not apply to these interfaces. In general, the appearance of interface states can be triggered by structural defects induced by geometrical reconstructions at the junction. However, with a careful choice of the systems in order to minimize the lattice mismatch and interfacial strain, as we have done in this work, we are confident that such defect-free interfaces are a good representation of the real systems.

On the other hand, we can clearly see in Fig. 3 that also the Schottky-Mott limit, where the SBH should be equal to the difference between the work function of the metal (difference between the Fermi level and the vacuum level) and that of the oxide (difference between the top of the valence band and vacuum level), does not hold either. In Fig. 3 we plot the $\mathrm{SBH}$ between $\mathrm{BaO}$ and various metals versus the work function of the metal. In the Schottky-Mott limit we would expect a linear relation between these two quantities, while our data are scattered without a precise relation. We can then conclude that for the interfaces considered, the SBH does not vary monotonically with the work function of the metal and in consequence does not obey the Schottky-Mott

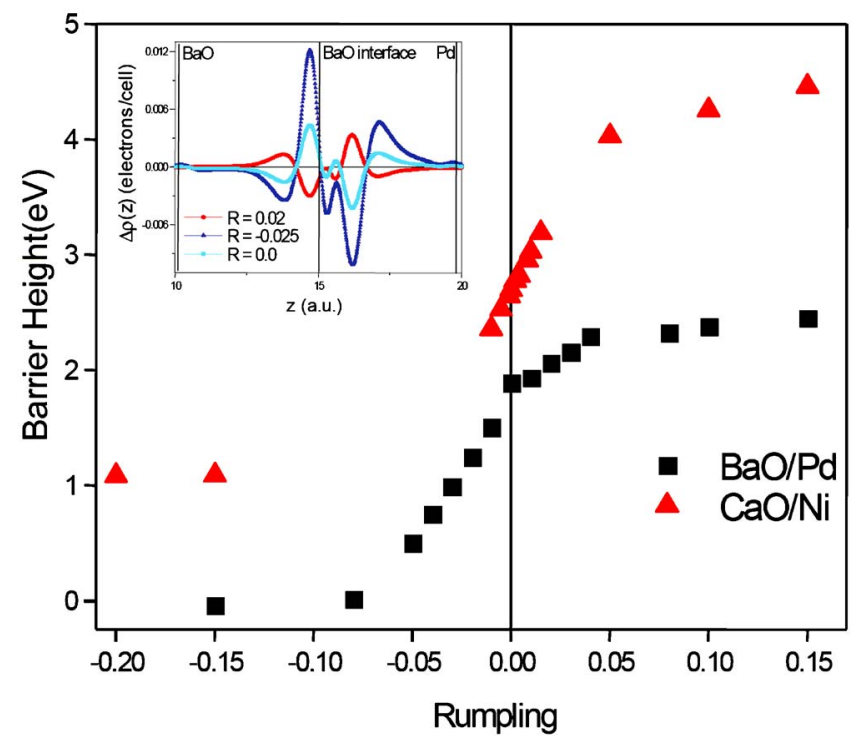

FIG. 4. (Color online) SBH vs artificial rumpling at the $\mathrm{BaO} / \mathrm{Pd}$ and $\mathrm{CaO} / \mathrm{Ni}$ interfaces. (inset) Lateral average of the electronic charge difference for three rumpling values: $0.02,-0.025$, and 0.0 in $\mathrm{BaO} / \mathrm{Pd}$. Very similar results are obtained for $\mathrm{CaO} / \mathrm{Ni}$. Vertical lines indicate the position of the different atomic planes as labeled in the picture.

rule. In all the interfaces that we considered, the SBH depends strongly on the details of the interfacial interactions.

The above results suggest that different hybridizations and consequent interface dipoles produce different band alignment for each individual case, and that the rumpling parameter seems to be an indicator of the strength of the interaction in the interface region, directly related to the amount of charge transfer that occurs at the interface. ${ }^{1}$ To isolate this effect, we simulated a $\mathrm{BaO} / \mathrm{Pd}$ and a $\mathrm{CaO} / \mathrm{Ni}$ interface where we artificially modified the rumpling by moving the atoms of the oxide interfacial plane and calculated the SBH for different rumpling values. The effect on the band alignment is shown in Fig. 4, where we plot the SBH as a function of the rumpling. Basically, by controlling the rumpling we can move the Fermi energy of the metal across the full LDA band gap of the oxides, confirming the prior observation that we do not see Fermi level pinning. At the same time, this is a very strong indication that the control of the rumpling is equivalent to the tuning of the SBH. In the inset of Fig. 4 we display the lateral average of the charge difference between the equilibrium configuration and selected configurations with the representative rumpling values of $0.0,0.02$, and -0.025 in the $\mathrm{BaO} / \mathrm{Pd}$ interface. It is clear that the rumpling affects strongly the charge transfer at the interface (opposite rumpling gives rise to charge transfer in opposite directions), and thus, ultimately, the macroscopic interfacial dipole.

In order to control the rumpling in a physical rather than artificial way, we performed another series of calculations where we chose $\mathrm{BaO} / \mathrm{Pd}$ as prototypical interface, and we introduced a different metal layer at the oxide-metal contact (technically, we removed the last Pd layer and replaced it with a different metal monolayer to have a final geometry such as $\mathrm{BaO} / \mathrm{METAL} / \mathrm{Pd}$ ). As we can see in Fig. 2, we are 


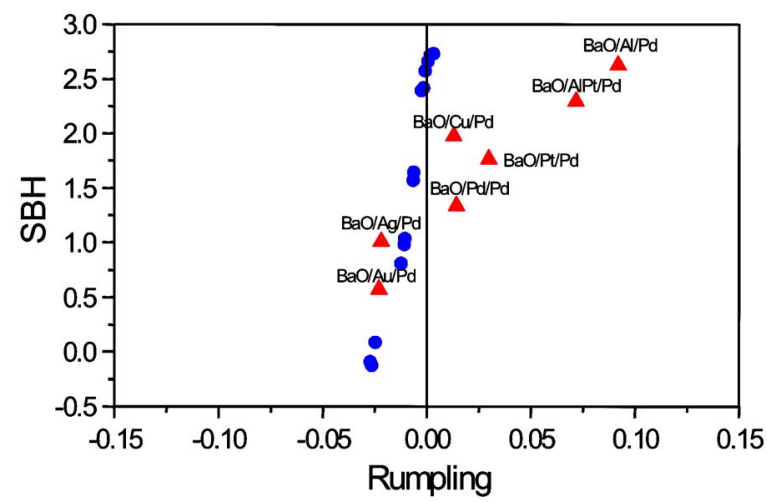

FIG. 5. (Color online) Comparison between the variation of the SBH induced by rumpling by the deposition of different metal layers at the interface of $\mathrm{BaOPd}$ (triangles), and by changing the interface distance between $\mathrm{Pd}$ and $\mathrm{BaO}$ (circles) via the application of an external strain. At each strain the system was fully relaxed, to obtain the corresponding value of the rumpling parameter.

able to tune the interface rumpling in a very efficient way, and span a broad range of SBH keeping the same oxide and external metal. We indeed show that one can either lower or increase the SBH with respect to the "clean" value just by changing a single atomic plane at the interface. Moreover, one can attain a continuous variation of the barrier height if metal alloys are considered. As an illustration, we computed the SBH for a 50-50 alloy of $\mathrm{Pt}$ and $\mathrm{Al}$, and found the corresponding value equal to the arithmetic average of the barriers for the pure metals.

Another important parameter that cannot be ignored is the interface distance, defined as the distance between two ideal planes that cut the interfacial planes of the two systems, av- eraged over the interface relaxations. The correlation between the rumpling and the interface distance is illustrated in Fig. 5, where we artificially changed the interface distance in the $\mathrm{BaO} / \mathrm{Pd}$ system, relaxed the atomic coordinates, and obtained the rumpling and SBH of this modified geometry. These results show very clearly that the interface distance and the rumpling parameter are only weakly related, and that they can be interpreted as two distinct ways of tuning the SBH. Interface distance can be partly controlled by an applied pressure, while the rumpling is determined by the chemical design of the interface layers.

\section{B. Electronic states and the modified Schottky-Mott rule}

The linear relation between the SBH and the rumpling parameter, confirmed also by results on interfaces other than $\mathrm{BaO} / \mathrm{Pd}$, suggests the possibility of an underlining rule $a$ la Schottky-Mott, to predict, given a metal and an oxide, what the SBH should be. We have already demonstrated that macroscopic concepts such as work functions and electron affinities will not work, since they do not capture the details of the microscopic interactions between the two materials. One logical procedure is then to analyze the local density of states (LDOS) of the interface layers, to gain more knowledge about the local electronic structure of the systems and their mutual relationship.

We chose to analyze four distinct contacts interfaced with $\mathrm{BaO}$ : Pd, our reference system, and Pd with different intralayers, $\mathrm{Cu} / \mathrm{Pd}, \mathrm{Pt} / \mathrm{Pd}$, and $\mathrm{Au} / \mathrm{Pd}$. This choice is motivated by the fact that the $\mathrm{SBH}$ of $\mathrm{BaO}$ with $\mathrm{Cu} / \mathrm{Pd}$ and $\mathrm{Pt} / \mathrm{Pd}$ is higher than with $\mathrm{Pd}$, while the SBH with $\mathrm{Au} / \mathrm{Pd}$ is lower. In Fig. 6 we report both the LDOS of the different interfaces (left panels), and the contour plot of the difference in the

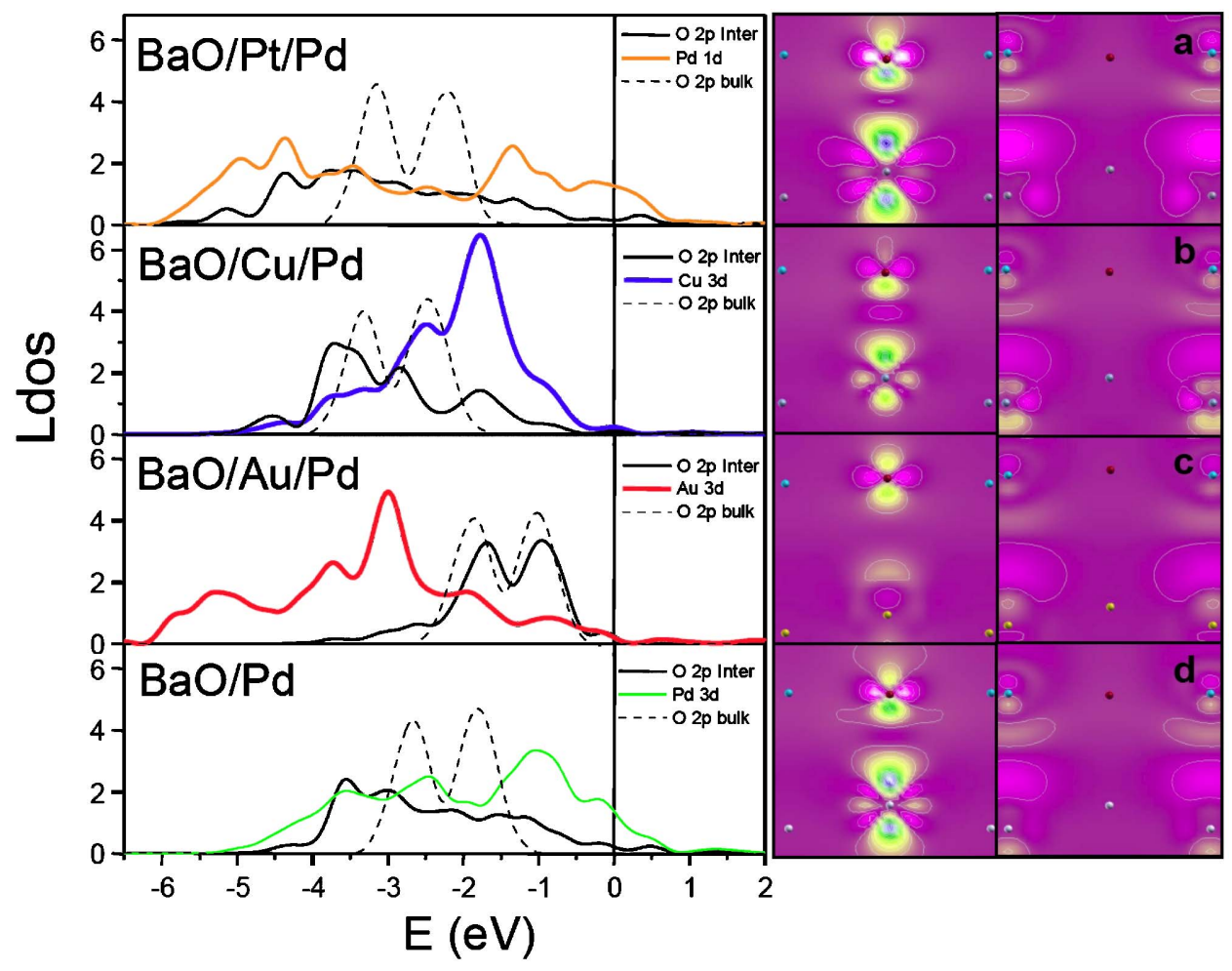

FIG. 6. (Color online) Left panels: LDOS of the $\mathrm{O} 2 p$ and metal $d$ orbitals at various metal/ $\mathrm{BaO}$ interfaces and, right panels: contour plot of the charge energy difference in the (001) plane passing through the metal-oxygen bond (left) and the metal-Barium bond (right) for four different interfacial systems: (a) $\mathrm{BaO} / \mathrm{Pt} / \mathrm{Pd}$; (b) $\mathrm{BaO} / \mathrm{Cu} / \mathrm{Pd}$; (c) $\mathrm{BaO} / \mathrm{Au} / \mathrm{Pd}$; (d) $\mathrm{BaO} / \mathrm{Pd}$. The zero in the energy scale is the Fermi level of the metallic slab. Contour lines are spaced 0.02 electrons/cell [yellow (light gray) = more negative, purple (darker gray) = more positive]. 


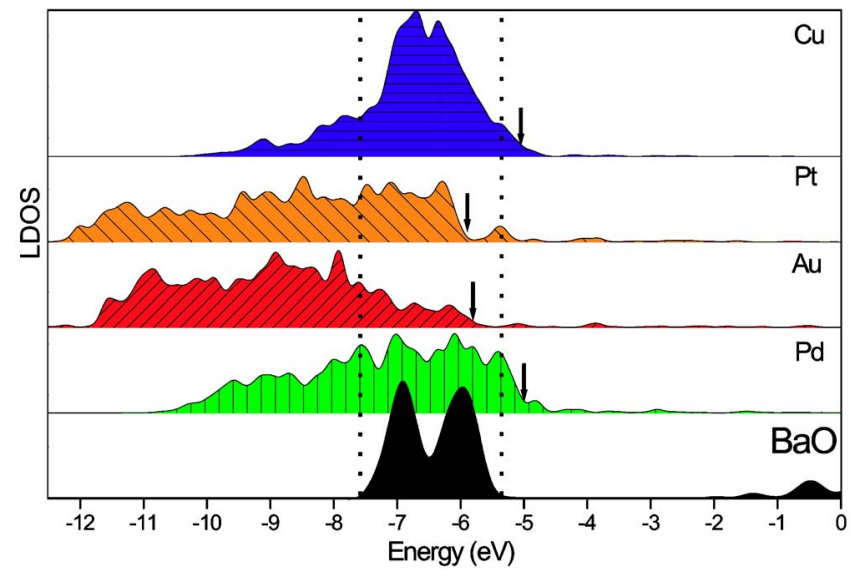

FIG. 7. (Color online) Surface LDOS of a monolayer of a metal $(\mathrm{Cu}, \mathrm{Pt}, \mathrm{Au}, \mathrm{Pd})$ on a Pd slab, compared with the surface LDOS of a $\mathrm{BaO}$ slab. The zero in the energy scale is the vacuum level and the arrows signal the Fermi energy levels. The metal slabs were fully relaxed.

electron charge density $\Delta \rho=[\rho(\mathrm{BaO} / \mathrm{Metal} / \mathrm{Pd})-\rho(\mathrm{BaO})-\rho$ (Metal/Pd)] (right panels), which gives us an indication of the charge transfer that takes place. In the LDOS plots, we show the three main contributions: Oxygen $2 p$ states of the bulk $\mathrm{BaO}$ and of the oxide interface layer, and the $d$ states of the first metal layer at the interface. Looking first at the $\mathrm{BaO} / \mathrm{Pd}$ interface [Fig. 6(d), left panel], we see that there is a significant change in shape of the oxygen $2 p$ bands of $\mathrm{BaO}$ going from the bulk to the interface layer. This indicates that the states of the oxide are strongly affected by the metal. The redistribution of charge at the interface can be seen in the charge difference plot [Fig. 6(d), right panel], which shows a transfer of charge from the $d_{z^{2}}$ to the $d_{z^{2}-y^{2}}$ orbitals of the Pd atoms bonded with the $\mathrm{O}$ atoms in the oxide. Very similar results have been reported ${ }^{9}$ for the $\mathrm{Pt}-\mathrm{O}$ bond at the interface between $\mathrm{BaTiO}_{3}$ and half a monolayer of Pt. At the same time also the oxygen electrons rehybridize and we observe a transfer of charge from the $p_{z}$ to the $p_{x y}$ orbitals. The redistribution of charge at the $\mathrm{Ba}-\mathrm{Pd}$ bond is minor and does not contribute much to the interface dipole.

With these results in mind, we can look at the effect of adding a layer of a different metal at the interface. Here, we observe that different metals can change dramatically the interface hybridizations and thus have a direct effect on the SBH. In particular we are interested in understanding the correlation between the relative position of the metal $d$ states and the oxygen $p$ states in the oxide. Starting with the interface $\mathrm{BaO} / \mathrm{Au} / \mathrm{Pd}$ [Fig. 6(c)], we observe that the overlap between the $d$ and $p$ states is much less significant than that for the $\mathrm{BaO} / \mathrm{Pd}$ interface, and that it is significantly shifted towards lower energies. As a consequence, the first layer of $\mathrm{Au}$ atoms does not hybridize with the $\mathrm{BaO}$ layer as strongly as in the case of the $\mathrm{Pd}$ atoms in the $\mathrm{BaO} / \mathrm{Pd}$ interface. The LDOS of the first layer of the oxide remains similar (except minor changes) to the LDOS of a layer of atoms deep in the bulk. Also, the LDOS of the Au interface layer remains similar in shape to that of the outer layer of the $\mathrm{Pd} / \mathrm{Au}$ slab.

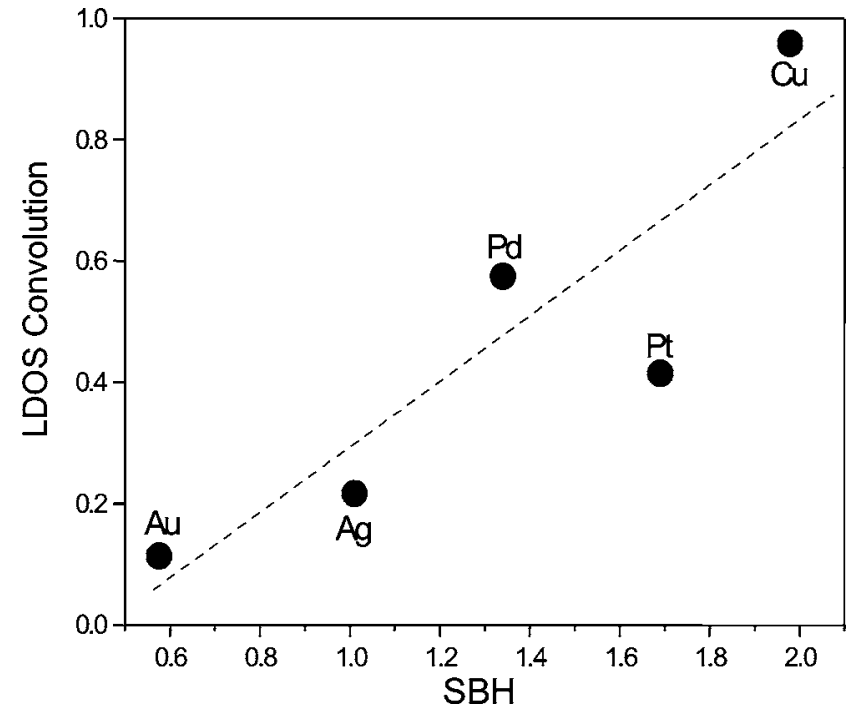

FIG. 8. Convolution of the LDOS of the $\mathrm{O} p$ and metal $d$ orbital vs the SBH.

These details are reflected in the charge transfer plots. The main difference with respect to the O-Pd bond is that the charge transfer between the $d_{z^{2}}$ states to the $d_{z^{2}-y^{2}}$ states is very small for the Au-O bond. This clearly affects the interface dipole through the rumpling of the oxide plane, which becomes negative, thus changing the sign of the interface dipole. This effect is responsible for the lowering of the SBH with respect to the pristine $\mathrm{BaO} / \mathrm{Pd}$ interface.

On the other hand, a metal that has a LDOS for the surface layer of the composed Pd/Metal slab shifted towards higher energies wrt. the $\mathrm{O} p$ states, as is the case for $\mathrm{Pt}$ and $\mathrm{Cu}$, will overlap differently with the first layer of the $\mathrm{BaO}$ slab [see Figs. 6(a) and 6(b)]. This will result in a strong hybridization whose main consequence is to push the oxygen atoms towards the metal slab, changing back the sign of the rumpling of the oxide plane, which is again positive. This, plus the relative position of the $d$ and $p$ states, produces a greater interface dipole, and consequently a higher energy difference between the oxide valence band and the Fermi energy of the metal.

From these data we can conclude that the intrinsic properties of the SBH between a metal and an oxide depend strongly upon the individual electronic structures of the two systems, and that there is no simple rule of thumb to deduce SBH from work functions or electron affinities. However, a careful analysis of the surface local density of states revealed a close relation between the relative overlap of the electronic states and the variation of the SBH.

In Fig. 7 we show the surface LDOS of a Pd slab both clean and terminated with a layer of another metal. In all cases, the slabs were carefully relaxed. Since the main contribution to the LDOS comes from the metallic $d$ states, we show only that contribution. As a reference, we also show the LDOS of the surface layer of a $\mathrm{BaO}$ slab where the main contribution to the valance band comes from the oxygen $p$ states. Energies are referred to the vacuum level, and we corrected the energy scale for $\mathrm{BaO}$ using the experimental value of the band gap ${ }^{5}$ and electron affinity. ${ }^{10}$ The arrows 
indicate the position of the Fermi levels for each case. If we analyze this data in terms of the overlapping of the $d$ states of the metal with the valence band of $\mathrm{BaO}$, we observe immediately that, taking the $\mathrm{Pd}$ as a reference, a higher overlap corresponds to a higher SBH. We can quantify this overlap by computing the convolution of the $\operatorname{LDOS}(d)$ and $\operatorname{LDOS}(p)$ between the bottom of the oxide valence band and either the metal Fermi energy or the top of the oxide valence band, whichever comes first. This is a rough measure of the available phase space for the electrons to redistribute the charge at the interface, and thus determine the relative rumpling. Of course, once the two surfaces are brought together, this initial overlap evolves into the true electronic interaction that determines the final SBH. The result of the above convolution is shown in Fig. 8, where the almost linear relation between the SBH and the convolution parameter is evident. We want to stress here that these data come from surface calculations, in the Schottky-Mott limit of an infinitely large interfacial distance where both systems have a common vacuum level. We can thus conclude by enunciating a "modified Schottky-Mott rule" for this class of metal-oxides interfaces: in the limit of surfaces infinitely apart and given a reference metal-oxide interface with a well defined Schottky barrier height and overlap convolution parameter, changes in the metal system will modify the relative overlap so that higher overlaps will increase the SBH and lower overlaps will decrease it.

\section{CONCLUSIONS}

In this work we have elucidated the role of different geometrical parameters in the tuning and control of the SBH in a class of metal-alkaline earth oxide interfaces. In particular, we have shown how one can tune the band alignment at the heterojunction by a selective control of the chemical species present at the interface. This manipulation has a fundamental quantum-mechanical basis, since ultimately we have shown how to finely control the charge rearrangement at the interface via the control of the heteroepitaxy at the substrate. Although we limited our investigation to a single class of systems, we believe that our results are prototypical of the behavior of a much broader collection of physical interfaces.

\section{ACKNOWLEDGMENTS}

The authors wish to acknowledge enlightening discussions with Rodney McKee, Fred Walker, Vincent Meunier, and Arrigo Calzolari. This work has been supported in part by: the National Science Foundation through Grant No. DMR-0304299; the Office of Basic Energy Sciences, U.S. Department of Energy at Oak Ridge National Laboratory under Contract No. DE-AC05-00OR22725 with UT-Battelle, LLC. Calculations have been carried out at the Center for Computational Sciences at ORNL, the NC State University HPC, and Grid Computing initiative.
${ }^{1}$ R. A. McKee, F. J. Walker, M. Buongiorno Nardelli, W. A. Shelton, and G. M. Stocks, Science 300, 1726 (2003).

${ }^{2} \mathrm{See}$, for instance, J. Goniakowski and C. Noguera, Interface Sci. 12, 93 (2004) and references therein.

${ }^{3}$ Electronic structure calculations have been performed using the PWscf package (S. Baroni, A. Dal Corso, S. De Gironcoli, and P. Giannozzi, http://www.pwscf.org). The electronic part is described by ultrasoft pseudopotentials [as in D. Vanderbilt, Phys. Rev. B 41, 7892 (1990)] where we have explicitly included as valence states the semicore $\mathrm{Sr} 4 s 4 p$ and $\mathrm{Ba} 5 s 5 p$ states as valence states. The exchange and correlation term is calculated within Local Density Approximation (LDA) [J. Perdew and A. Zunger, Phys. Rev. B 23, 5048 (1981)]; D. Ceperley and B. Alder, Phys. Rev. Lett. 45, 566 (1980)]. Tests using functional with gradient correction did not show significant quantitative differences. The reciprocal space integrations were performed on a (661) Monkhorst-Pack mesh using a smearing parameter $\sigma$ $=0.01 \mathrm{Ry}$.
${ }^{4}$ S. Baroni, M. Peressi, R. Resta, and A. Baldereschi, in Proceedings of the 21st International Conference on the Physics of Semiconductors, edited by Ping Jiang and Hou-Zhi Zheng (World Scientific, Singapore, 1993), p. 689; A. Ruini, R. Resta, and S. Baroni, Phys. Rev. B 56, 14921 (1997).

${ }^{5}$ Due to the well-known underestimation of the energy gap in DFTLDA, throughout this paper we will use the experimental gap energies for the oxides when needed [see, for instance, Defects and Their Structure in Nonmetallic Solids, edited by B. Henderson and A. E. Hughes (Plenum, New York, 1976)].

${ }^{6}$ J. Bardeen, Phys. Rev. 71, 717 (1947).

${ }^{7}$ N. F. Mott, Proc. Cambridge Philos. Soc. 34, 568 (1938).

${ }^{8}$ W. Schottky, Phys. Z. 113, 367 (1940).

${ }^{9}$ F. Rao, M. Kim, and A. J. Freeman, Phys. Rev. B 55, 13953 (1997).

${ }^{10}$ K. Y. Tsou and E. B. Hensley, J. Appl. Phys. 45, 47 (1974).

${ }^{11}$ N. W. Ashcroft and N. D. Mermin, Solid State Physics (Sounders College, Philadelphia, 1974). 